La Cour de P'aris a donné à la question une solution idenlique daus un arrêt du a avril rgos.

IV. T..., licencié de B..., entendait prononcer la résiliation de son contral au jour du jugement et à ses torts el griefs jar jugement du i 2 janvier rgo6. Ia Cour de Paris confirmail le jugement par arret du 14 juin rgob qui déclarait to contral résilié du jour de l'arrêt.

Arant de faire signifier l'arrêt, B... alléguant que T... conlintail à se compoter on licencié, lajsail saisia au mois de juin 1906 un certain nombre d'objels qu'il prélendait conliefails et poursuivait ' $T$... ch contrefaçon.

Le Tribunal rejclait la demande en contrefagon el com meltait un expert pour établir le chiffre des redevances dues far T. . au 6 aont Igo6, jom de la significalion de l'arrêt.

sur appel de B..., la Cour confirmait le jugement par un arêt dont lo considérant suivant résume la situation des parlies en pareille circonstance :

"Considérant que le contrat de licence dont lexécution comporte des prestations périndiques correspondant ì Ja jouissance du brevel est un conlual sucessif . que tant quil n'a jas ćté annulé avant le terme fixé aux conventions, par me décison judiciaire définitive, il conlinue à exister ; que pendant l'instance d'appel, le licencie dont le comlall de lirince a ćlé amnulé au cours de son exploitation effective, peut, sans être contrefacleur, poursurve celte cécution dans les condilions prévues au contral, que, survenant un arêt confirmalif, celle décision n'a pas pour conséquence de supprimer les faits matériels de jouissance qui onl élé accomplis au cours de la procédure d'appel el que le licencić avait le droit de fairen.

Le licencié peut jouir du brevel d'une façon différente de celle prévue au contrat ot outrepasse les droits qui lui sont concédés - il devient aloes contrefacteur.

Telle scrail la situation du licencié qui, ayant une licenco de fabricilion on une licence de vente pour le départentent dı Rhòne, fabriquerait ou vendrait en dehors du département, du licencié qui, ayant une licence de fabrication, se liverail à la vente, ou, inversement, du licencié qui exploilerait après le torme fixé au contrat ou après sa résiliation.

(A suive).

Amédće Bugraxp.

Avocat à la Cour d'Appel de Lyor.

\section{DISTRIBUTIONS D'ÉNERGIE}

\section{ÉTUDE SUR LES DISJONCTEURS A COURANT CONTINU BASSE TENSION}

D'une manière génćrale, on appello disjoncteur un interrupteur destinć à ouvrir, automatiquement, un circuit lorsqu'il y a un inconvéniont ou un danger à le maintenir fermó.

si lo circuit doit être préservé contre un courant exagéré provenant d'une surcharge ou d'un court-circuil, on place In disjoncteur à maxima qui devra fonctionner chaque fois que l'iutensité dépassera une valeur déterninéc.

Si, au contraire, le circuit doil être ouvert à la suite d'une diminution d'intensilé ou d'une baisse de tension, on emploie, lans ce cas, un disjoncleur dit à minima. Ce disjoncleur devra fonctionner, par cxemple, lorsque le courant se lrouve accidentellement supprimé sur un réseau, afin de préserver les molcurs qui absorberaient une intensilé exagér'e à la remise sous tension du réseau, ou bien il pourra être

employé pour interrompre le coumant de charge d'une bat. terie d'accumulateurs lorsque celni-ci devient trop faible.

Enlin, si l'on veut évito un changement de strus du eoutant dans un circuil, comme le cas peut se produire avec ume batleric d'accumulateurs susceptible de se décharger dans les génćntrices, on place un disjoncleur à relour de rouramt.

Tous ces disjoncteurs, dans leur forme la plus simple, ou, vrent, presque instantanement, le circuil dès que vient à se

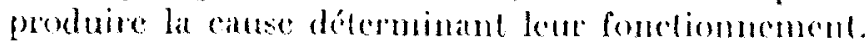

Or, souvent l'ouverture du circuit n'a pas bersin d'être effectuce aussi rapidenent el, si la perturbation dangereuse ne ture que peu de lemps, il cot le plus souvent inulito d'interrompre, pour une fausse alerte, la marche des mac aines ot d'apporter un tromble au foncliomement do loule mo inslallation. Pour ćvifer cet inconvénient, on a cré des disjoncleurs dits temporisés, qui sont disposés de façon ì n'ouvrir le ciscuit qu'après un lemps délermine ot dans le cas seulement où la cause de leur fonctionnement se maintient pendant toul ce temps. On a crée, égilcment, des apparcils encore plus parfails qui savent, on fueleque sorte, apprécice à quel moment ils doivent agir, ouvant lo circuit immedialement, si le danger est trop grand, ou allendant un lemps variable suivant limportance de la perturbalion. Ce sont les disjoncleurs à action retardée c à action diffirsé.

D'autre part, on emploir, de pas en plus, depuis queliues années, des disposilifs empechant de mantenir fermés les disjoncteurs tant que la cause qui doit les fairo fonctionner n'a pas disparu. les disjoncleurs soml, dans ce cas, dits à renclanchement empeché.

Dans celle élude, nous voulons, surtoul examinor le principe des disjonctens à minima, à maxima el à retour de courant, employés arec le coumant continu à basse lension at indipues égakement de quelle facon somt élablis, ordinairement, les disjoncteurs temporisés ou a action retardéc

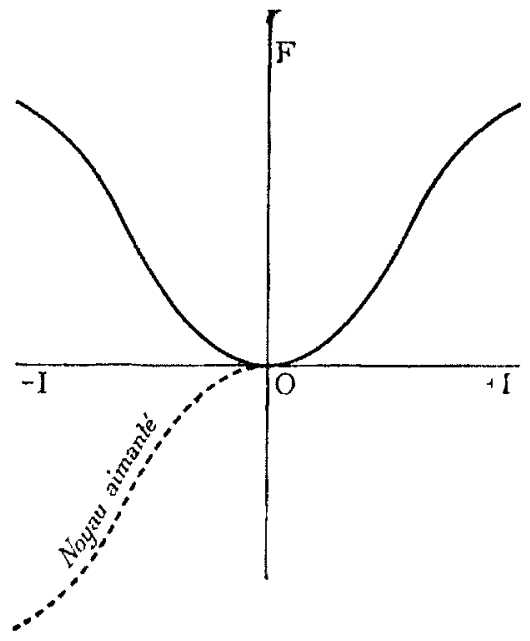

Fig. 1. - Courbe de l'efforl agissant sur une armalure en fonclion de l'inlensilé circulant dans le solénoïde. saml sur latmalure en fonclion do Jintensité circulant dans l'électro-aimant ou te solénö̈de.

On sait que d'une façon générale la force portante d'un électro-aimant est domóc par la formule suivante :

$$
F=\frac{B^{2} S}{8 \pi} \text { (dynes) }
$$

$B$ étant linduclion en Gauss ; $S$, la section du noyau en $\mathrm{cm}^{2}$;

$$
\begin{array}{lc}
\text { O' : } & S=\text { constante }=K \\
\text { D'où: } & F=K B^{2}
\end{array}
$$


L'induction $B$ étant proportionnelle à l'intensité $I$ dans la permière région de la courbe de magnétisme, on peut donc écrire :

$$
F=K^{\prime} l^{2}
$$

In courbe de $F^{\prime}$ en fonction de $l$ est donc une parabole à axe vertical qui s’incline légèrement vers l'axe des intensités à cause de la saluration du noyau (fig. I). Le noyau étant en fer doux, mélal ne conservant pas, ou peu, son aimantation, si le courant s'inverse la courbe des efforts reste toujours siluée en dessus de l'axe des $I$, el elle est symétrique pax rapport à l'axe des $F$. Cictle courbe est la même pour tous les solénoides avec novau en acier doux. Si Je noyau était un iimant, la courbe changerail de signe pour un courant inverse.

Disjoxcteur a minma simple. - Dans ces disjoncteurs, l'armature ou noyau est, en temps normal, colléc aux pôles de l'électio-aimant el ne s'en détache que lorsque le courant dans le solénoïde devient assez faible pour occasionner le fonctionnement de l'appareil.

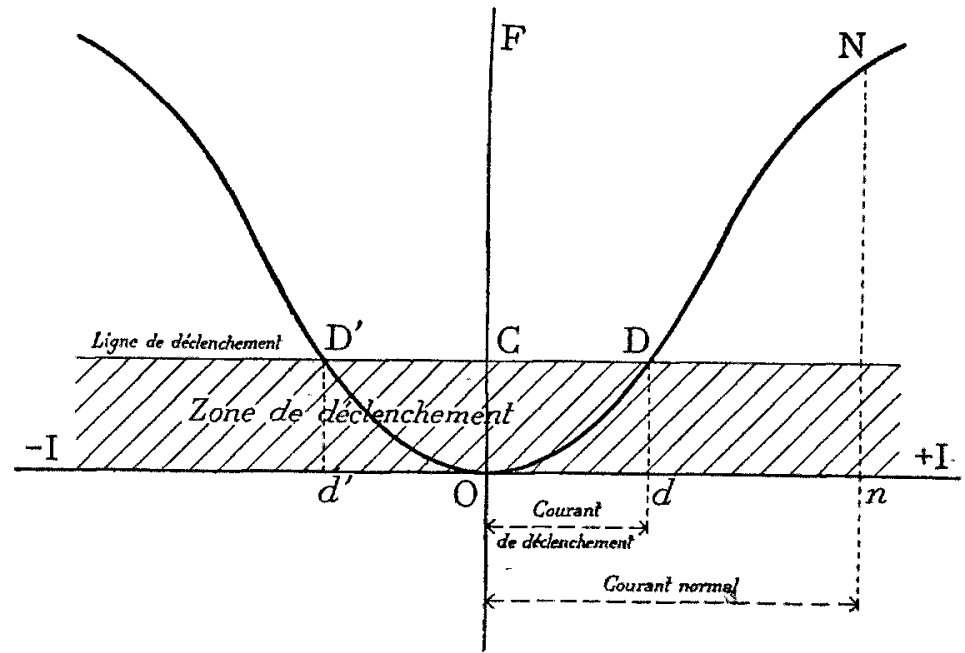

Fig. 2. - Caractéristique d'un disjoncteur à minima simple.

la Figure a représente la caractéristique d'un disjoncteur a minina simple.

Soit $O C$, l'effort nécessairc pour maintenir le noyau collé, ou en équilibre dans un certain cntrefer. La courbe des efforts coupe la droite parallèle à l'axe des abscisses menée par $C$ en deux points $D$ et $D^{\prime}$, symétriques par rapport à $O F$ et correspondant à deux points de déclanchement du disjoncloul : l'un $D$ pour un courant d'un cerlain sens, l'autrc $D^{\prime}$ pour le courant inverse de celui-ci.

Si $O d=$ courant minimum de disjonction,

On $=$ courant normal dans le circuit.

l.e rapport : $\frac{O d}{O n} \times 100=$ valeur du déchanchenent con $\%$ du courant nolmal.

En général, ce rapport est égal à ro, el souvent il est réglable de 7 à $20 \%$.

Nous avons dit que les disjoncteurs à minima devaient protéger les installations, où ils sonl employés, soit contre une baisse momentanće de la lension, soit comme dans le cas de la charge d'une ballerie d'accumulateurs, contre une charge de trop longue durée avec un courant de faible inLensité.

Dans le prenier cas, la bobine du disjoncteur, cnroulée avec du fil lin, est placéc en dérivation (fig. 3), et dans le second cas, elle est disposée en séric et ne pessède alors que peu de spires d'un conducteur à forte section (fig. 4).
Lórsque la bobine est en dérivation, il faut avoir soin de brancher celle-ci de façon quelle soit mise hors circuit après le fonctionnentent du disjoncteur.

En nous reportant à la courbe des efforts magnétiques (figure 2), l'on voit que si le courant s'inverse brusquenchl de $+O d$ à $-O d$, le disjoncleur peul rester enclançlić, malgré le passage à zéro de l'intensité. Ceci provient de ce que les efforts magnéliques, par suite de l'hystérésis, ne suivent pas, instantanément, les valcurs des courants qui les produisent.

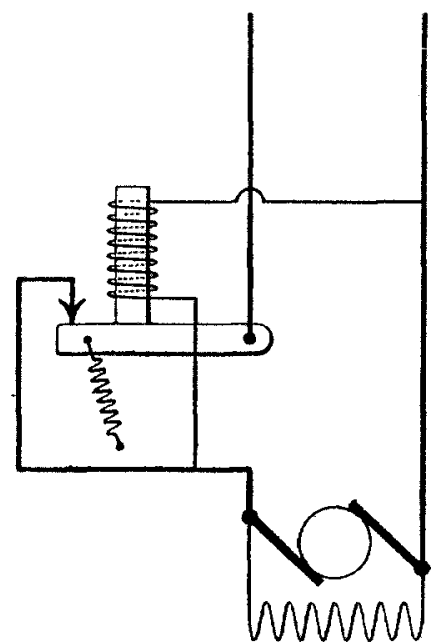

FIG. 3. - Disjoncteur à minima de tension

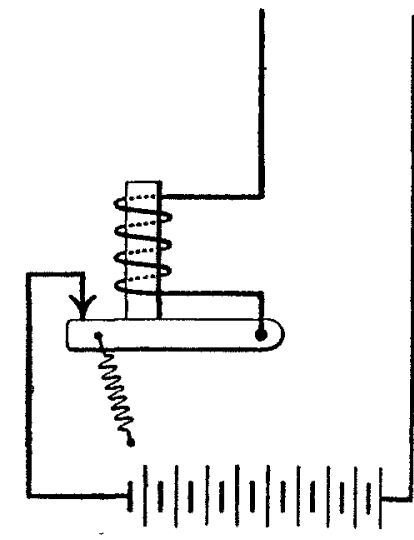

Fic. 4. - Disjoncteur a minima d'inlensité
Lorsque l'on a à craindre que le courant puisse changer rapidement de sens sur un réseau, on emplote, dans ce cas, un disjoncleur à minima polarisé.

Ce disjoncleur se différencie du précédent simplement cn ce que le circuit magnétique n'obéit pas, exclusivement, aux variations de la valcur des ampères-tours de la bobine et qu'il est influencé par le changement de sens du courant. Io procédé généralement employé pour obtenir cette condition consiste à introduire dans le circuit magnétique une bobine, mise en dérivation sur un circuit quelconque et produisant un flux fixe ou peu variable et indépendant du premier.

DISJONGTEUR a MINIMA POIARISÉ ET A INVERsion de cotRANT. - Quand, un tableau est alimenté par plusieurs feeders en quantité, les disjoncteurs, par inversion, sont plus avantageux que les coupe-circuits ou les disjoncteurs de surcharge (à maxima).

Les premiers séparent le fecder avarié du circuit avant qutc le courant soit devenu anormal et entrainent ainsi le minimum de perturbations. Les seconds, au contraire, menacent de mettre hors-circuil, non seulement le cáble défectuenx, mais tous les freder's sains el leur fonctionmenent nécessile un courant dangcroux.

Les conditions que doit remplir un bon disjoncleur à minima polarisé sont les suivanles :

$I^{\circ}$ Il faut qu'un comrant inverse égal à $25 \%$ du courant normal fasse fonctionner l'appareil.

$2^{\circ}$ L'effort magnétique doit conlinuer ì croître en mène temps que le courant inversé, de façon que toute paresse du mécanisme ail pour conséquence seulement de nécessiter, pour la rupture, un courant un peu plus grand et que, si lo courant inversé alteint une valcur élevée, le fonctionnement du disjoncteur n'en soit que plus certain.

$3^{\circ}$ Un accident à l'cnroulement fil fin ne doil pas fair fonctionner le disjoncteur.

Pour que l'on puisse oblenir un point de déclanchement a minima, il faut que la ligne de déclanchement soit siluéc au-dessus de la ligne des efforts shunt (fig. 5). En général, 
ces disjoncteurs sont réglés pour déclancher avec un courant minima ćgal à $\$ \%$ du courant normal, el un courant de retour de $15 \%$.

Je réglage ne peul pas se faire indépendamment à minima el en retour. Si l'on règle le disjoncteur pour fonctionnct à minima par exemple, la courbe des efforts combinćs nous montre qu'à un point de déclanchement $D$ à minima correspond un seul poinl Dr en retour.

Il n'y a pas de méthode bien détcrminée pour calculer de tels disjoncteurs, c'est une question d'essais au laboratoire et lorsqu'une série est bien ćtablie, et qu'on a soin de relever les caractéristiques principales, il est facile de déterminer, par comparaison, les autres types demandés par les clicnts.

Sur la figure 5, la courbe $E$ représente la courbe des efforts dûs à la bobine principale. La droite $G$ correspond à l'effort constant de l'enroulement fil fin. En combinanl les efforts dûs à l'enroulement principal branché en série el à l'enroulcment en dérivation on obtient l'effort résultant agissanl sur l'armalure et représenté par la courbe $F$.

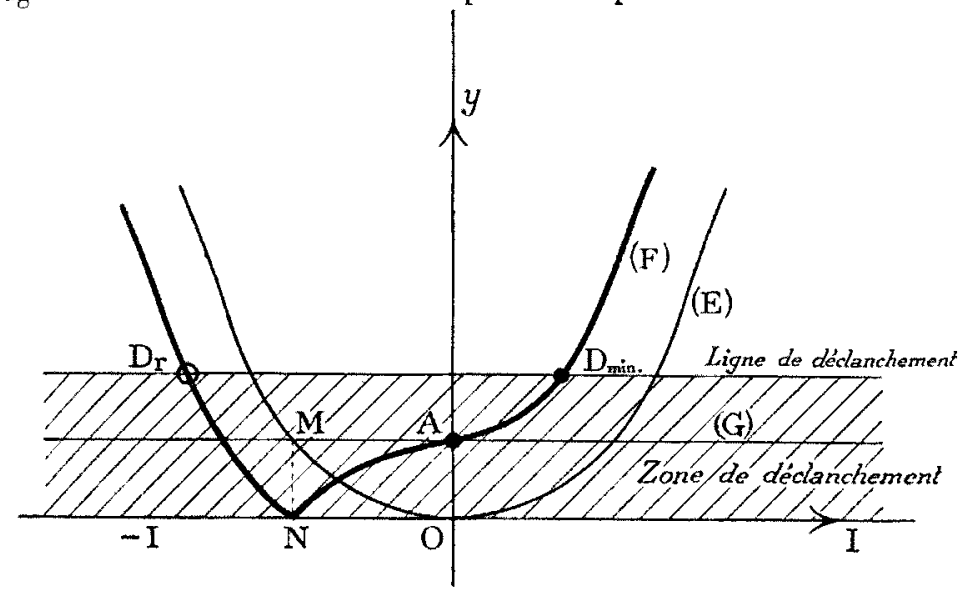

Fig. 5. - Caracléristique d'un disjoncteur minima polarisé et à inversion de courant

Lorsque le comrant s'inverse, l'effort produil par l'enroulement-série se retranche de l'effort dù au shunt. En $M$, l'effort série cst égal à l'effort shunt, on obtient un point $N$ de la courbe $(F)$ sur l'axe $O I$; au-delà du point $M$, l'effort série devient prédominant.

On voit que la courbe $F$ coupe la ligne de déclanchement en deux points $D_{\text {min }}$ et $D_{r}$ donc, si le courant inverse at. teint brusquement, une valeur supérieure à $D$, le disjoncteur ne fonctionne pas. Il est à remarquer aussi que si l'enroulement shunt est coupé, l'appareil fonctionne.

Ce disjoncteur ne répond donc pas complètement aux conditions énoncées précédemment.

Un procédé simple consiste à réaliser l'armalure mobile du disjoncteur par un morceau d'acier aimanté. Cette armalure, si le courant principal vient à cesser, maintient lc disjoncteur enclanché ; mais si le courant change de

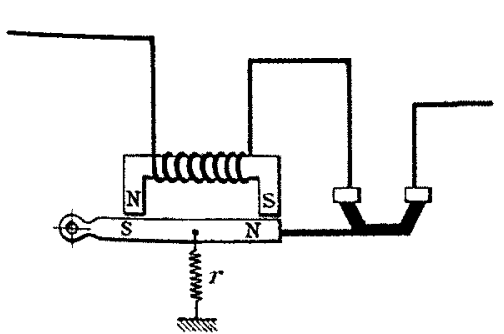

FIG. 6. - Disjoncleur ì minima polarisé sens, le flux magnétique tendant à être inversé, le barreau d'acier se désaimante el lorsque son magnétisme devient nul (avant de prendre un sens inverse), le disjoncleur déclanche (fig. 6). Ce genre de disjoncteur polarisé est le plus simple, mais il n'est pas applicable dans tous les cas. L'armature d'acier exige, cn offel, pour sa polarisation une intensité relative- ment forte, el si le déclanchement se fait strement lorsquil y a inversion du comant, il faul pour cela un lones appreciable el une intensite de sens contraire assez forte.

Disjoxctecr a maxima simple. - Les disjoncteurs à maxima sont des appareils qui rompent le circuit lorsque le courant dépasse une intensité déterminée. Ils protègent done les installations contre los surcharges el les courts-circuils.

le point de déclanchement de ces appareils esl en général réglable à volonlé depuis ro o jusqu’à roo \% de l'intensilé nor' male du circuil. L'intensilé normale est celle pour laquelle sont calculées les différentes pièces de l'apparcil pour avoir un échauffement convenable.

La figure 7 représente le schéma d'un lel disjoncleur. La bobine $B$, lorsque l'intensilé est trop forte, attire son armature el fait soulever le crochet qui maintient fermé l'interrupleur.

Le réglage du point de déclanchement s'obticnt en faisant varier, à l'aide d'un écrou et contreécrou, la position du noyau $n$, par rapport à la bobine $B$.

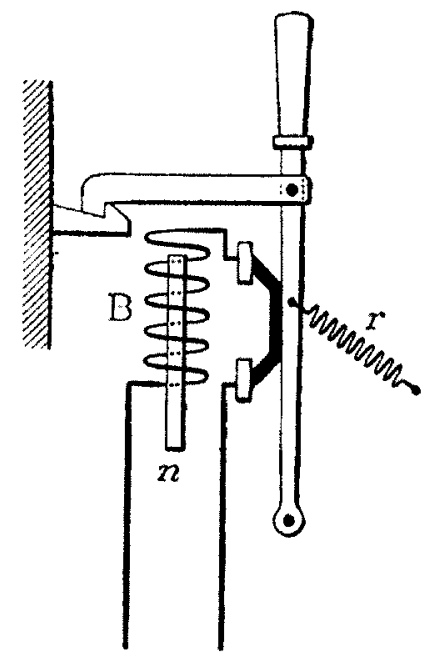

Fis. $\%$ - Disjonclem it maxima
Dans ce disjoncteur, la bobine $B$ est parcourue par l'intensité tolale du circuit, lorsque cette intensité devient trop grande, il est préférable de placer cetle bobine en dórivation sur un shunt avec une résistance en série pour permellue le réglage.

Caracténisteque doun disjoncteur a maxima. - Ia figure 8 représente la courbe des efforts agissant sur le noyau en fonction de l'intensité du courant dans le circuit. Celle courbe des efforts coupe la ligne de déchanchement $\mathrm{mm}$ en deux points $D$ symétriques par rapport à l'axe $O F$. Ces deux points correspondent, l'un à un courant direct, l'aulre

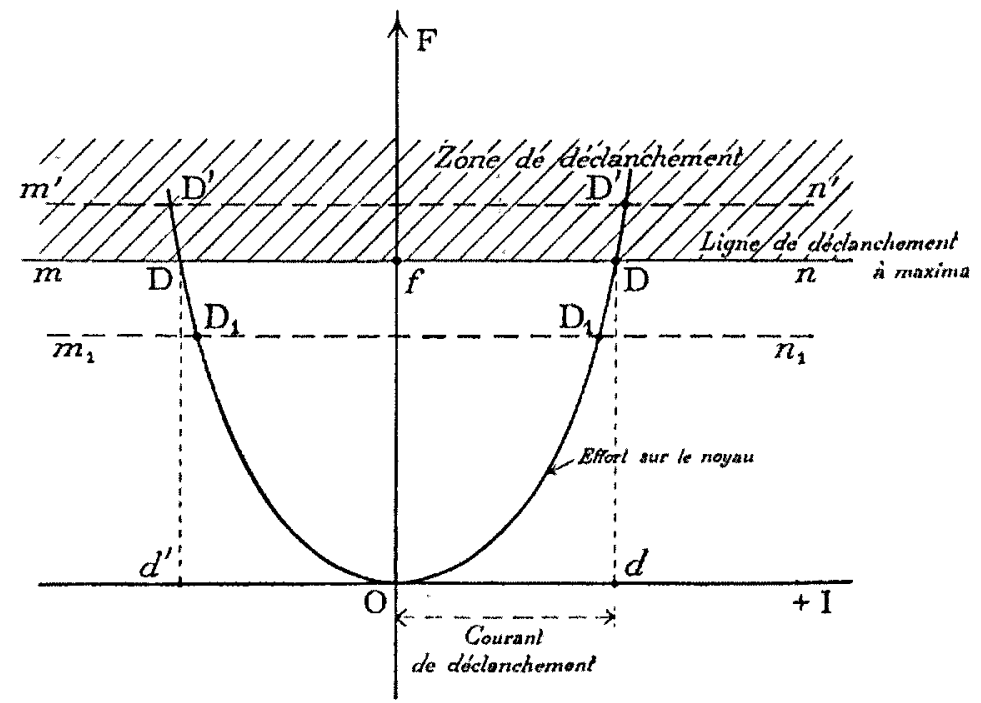

Fic. 8. - Caractéristique d'un disjoncleur à maxima

à un courant inverse. Lorsqu'on fail varier, comme on l'it indiqué précódemment, la position du noyau par rapport à la bobine, c'est-à-dire la valeur de l'entrefer, on déplace: la ligne de déclanchement mn parallelement à clle-meme en ' $m$ 'n' ou $m_{1} n_{1}$ et l'on obtient ainsi un déclanchement d: l'appareil pour des intensités plus rapprochées ou plus éloi. gnées de l'intensité normale. 
Disjoncteur a Maxima et a jetodr de conrant. - Ces disjoncteurs ont pour but de protéger les appareils contre les courts-circuits et les surcharges, et en meme temps contre les inversions de courant qui peuvent être dangereuses. Le champ magnélique est créé par deux enroulements concentriques dont l'un est en série dans le circuit et l'autre en dérivation.

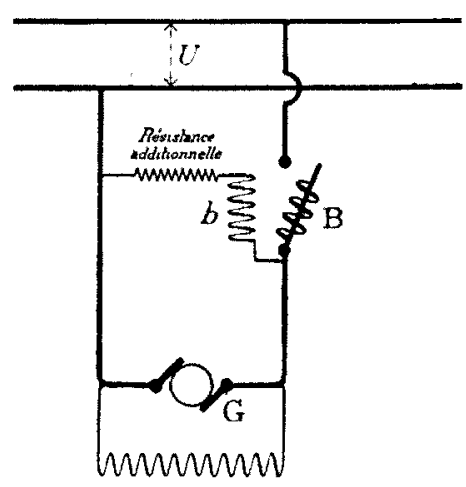

Fic. 9. - Emploi d'un disjoncleur à máxima et à relour de courant dans le cas d'une généralrice.

Les enroulements de ces deux solénoüde doivent être connectés de telle façon qu'cn fonctionnement normal les flux produits par ces deux bobines soient opposés l'un à l'autre.

En cas d'inversion de courant, le sens du courant dans la bobine en dérivation ne changeant pas, les actions des deux bobines s'ajoulant déterminent le soulevement du noyau mobile, qui, par son choc, proroque l'ouverture du disjoncleur.

Dans tous les cas, le montage doil être effeclué de lelle facon que le circuit de la bobine fil fin cesse d'élre alimenté lorsque le disjoncteur a fonclionne.

Supposons une génératrice débitant sur un réseau et un disjoncteur à maxima et à retour de courant monté comme lindique la figure $g$.

Lorsque la f. e. m. de la génératrice devient supéricure à la teusion $U$ du réseau, elle débite sur celui-ci.

Si au contraire la $\mathrm{f}$. e. $\mathrm{m}$. de la génératrice devient infericure à la tension du réseau, on a un retour de courant et la génératrice lend à fonctionner en moteur.

Si Rd représente la résistance de la bobine fil fin et la résislance additionnelle, le courant dans la bobine fil fin est sensiblement conslant et a pour valeur :

$$
I d=\frac{U}{R d}
$$

Désignons par :

nd le nombre de spires de la bobine en dérivation,

ns le nombre de spires de la bobine en série ;

Is le courant fourni par la génératrice.

Ir le courant le relour.

Nous savons que les ampères-tours fil fin se retranchent des ampères-tours série dans le sens direct et s'ajoutent en retour de courant.

Si 1 est le nombre d’ampères-tours nécessaires pour produire le déclanchement, on a les deux équations do fonctionnement :

Dans le sens dirèct:

En retour de courant :

$$
\begin{aligned}
& n_{s} I_{s}-n_{d} I_{d}=A . \\
& n_{s} I_{r}+n_{d} I_{d}=A .
\end{aligned}
$$

D'où : $\quad I_{s}-I_{r}=\frac{2 n_{d} I_{d}}{n_{s}}=$ constante.

Is et $I r$ sont les deux intensilés qui font fonctionner le disjoncteur soil à maxima soit à relour de courant.

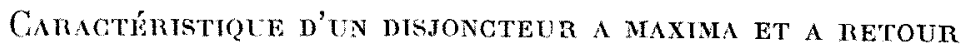
DE Covnanr. - La représentation graphique du fonctionnemenl d'un disjoncteur à maxima et à retour de courant se fait de la mème façon que dans le cas d'un disjoncteur à minima (fig. ro). On trace la courbe des efforts dus à l'en- roulement série, la droite représcntant les efforts constants produits par la bobine shunt et la ligne de déclanchement. Pour des intensités positives, la différence des efforts série of shunt sera $a d-a b=a c$.

L'effort résultant sur l'armature diminue d'abord jusquà ce que les ampères-tours de l'enroulement série deviennent égaux à ceux de la bobine shunl, en $E$ l'effort résultant est nul, puis cet effort augmente ensuite constamment.

Le point d'intersection $D_{m}$ a de la courbe des efforts com. binés avec la ligne de déclanchement correspond au point de déclanchement à maxima.

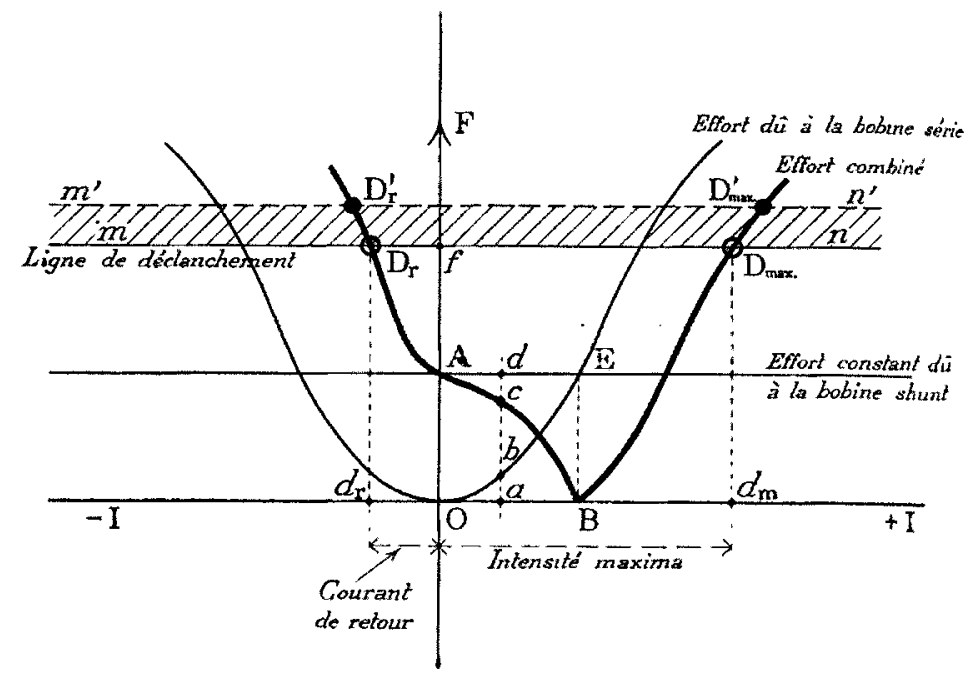

F1G. Io. - Caraclérislique d'un disjoncleur à maxima el à relour de courant.

Lorsque le courant s'inverse, les efforts s'ajoutent, la courbe partant de $A$ monte constamment et l'on obtient en $D_{r}$ le point de déclanchement par retour de courant.

Limites de rúgrage. - Ces appareils sont habituellement réglés pour déclancher dès que le courant atleint une intensité négative d'une valeur égale à $10 \%$ du courant nor. mal, ou bien pour un courant direct dépassant de $50 \%$ l'intensité normale. Si l'on augmente l'entrefer de la bobine, on écarte simultanément les deux limites, car, sur notre graphique, toute augmentation d'entrefer correspond à un déplacement de la ligne de déclanchement.

Ia ligne de déclanchoment peul venir en m'n', par exemple, ce qui donne deux nouveaux points de déclanchement à maxima et à retour de courant $D^{\prime}$ max. et $D^{\prime} r$.

Ainsi, par exemple, un disjoncteur de a ooo ampères, inlensité normale, réglé à $55 \mathrm{~mm}$. d'entrefer, déclanchera à maxima pour un courant direct de I 500 ampères et par inversion de courant pour roo ampères; avec un entrefer plus plus grand le déclanchement se produrait, par exemple, ì $1550 A$ et à $150 A$.

La différence entre les deux intensités de déclanchement resle toujours constante, quel que soit l'entrefer.

Si l'on désirait régler indépendamment ces doux limites, il serait indispensable d'employer un disjoncteur à maxima et minima polarisé.

Il est à recommander de ne pas donner à l'entrefer une valeur trop inféricure à $15 \mathrm{~mm}$. pour éviter que le choc áu noyau sur le crochet ne devienne insuffisant pour faire diclancher l'appareil.

Disjoxcteur a maxima et a minima polarisé. - Nous venons de voir que dans un disjoncteur à maxima et à retour de courant, la différence existant entre les deux intensités de déclanchrment (déclanchement à maxima et à retour de 
courant) est constante, cl que, par suite, il est impossible de régler indépendamment les deux valeurs de l'intensité de déclanchement. Lorsqu'on veut rendre indépendants les deux courants de déclanchement, on emploie un disjoncteur i maxima el à minima polarisé (figure ar). Cet appareil se compose de deux bobines agissant sur le mê-

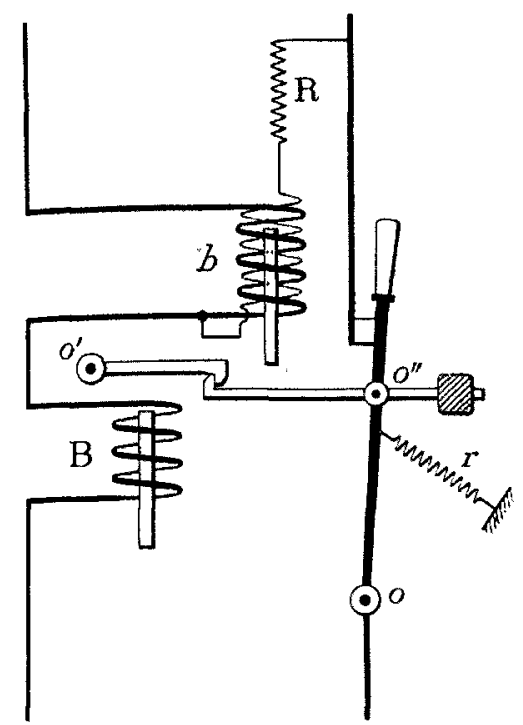

Fıg. I I. - Disjoncteur à maxima el à minima polarisé. me mécanisme, l'une $B$ à maxima, l'autre $b$ à minima polarisé. Le noyau de la bobine à minima agit par sa chute et celui de la bobine à maxima par choc au moment de son soulèvement. Le point de déclanchement à maxima peul être réglé en faisant varier l'entrefer de la bobine $B$ (comme dans un disjoncleur à maxima simple), et celui à retour de courant peut être déterminé une fois pour toules, sans relation avec celui à maxima. On a, en somme, deux appareils différents, l'un à maxima simple (caractéristique, fig. S), l'aulre à retour de courant (caractéristique, ligure 5).

C's disjoncteurs sont surtout employés pour les génératrices destinées à la charge des batteries d'accumulateurs.

Disjoncteurs a maxima et a Minima pour faibles intensnés. - Lorsque le courant normal ne dépasse pas 60 ampères environ, on emploie comme disjoncleur à maxima et à minima l'appareil représenté par la figure rs. Cel apparcil esl composé de deux bobines, l'une à maxima $B$, l'autre à minima $b$, placée en dérivalion sur une résistance $R$. La bobine $b$ attire une armaturc $p u$, qui ferme lo circuit en ef. Si le courant devient exagéré, la bobine $B$ attire son noyau qui, venant en conlact avec los points $a$ ct $c$, met la bobine $b$ cu court-circuil.

Lorsque le courant est trop faible dans le

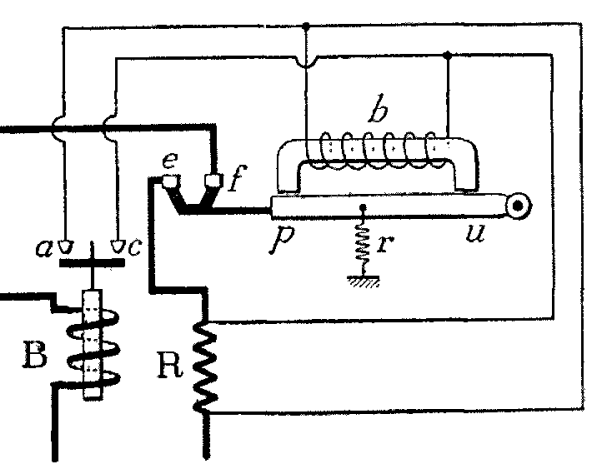

Fig. 12. - Disjoncleur à maxima el à minima pour faible intensité.

circuit, la chute de potentiel $R I$ dans la résistance $R$ n'est plus suffisante poux entretenir le courant convenable dans la bobine $b$ et l'appareil déclanche.

Lc réglage peut s'opérer à maxima par la position du noyau dans la bobine $B$ et à minima par la valeur de la résistance $R$, ou bien, à la rigueur, par la tension du ressort.

J.es disjoncteurs à maxima et à minima se font généralement :

I A maxima et à minima d'intensité ;

$2^{\circ}$ A maxima d'intensité et à minima de tension.

I.es caractéristiques sont celles d'un maxima et d'un minima.

Disjoncteurs teuporisés. - Les disjoncleurs à maxima sont destinés à remplacer de plus en plus les fusibles, mais

ils ont, sur ces derniers, l'inconvenient de fonctionner parfois trop rapidement. Un fusible préscnle, en effet, une certaine inertie; si le court-circuit ne dure qu'un temps tres court, il peut n'avoir pas d'inconvénients, le fusible n'aura pas le temps de fondre, mais un disjoncteur, placé dims les mêmes condilions, fonctionnera.

Lorsqu'on reut éviter le fonctionnement intempestif du disjoncleur, par suite d'un à-coup momenlané ou d'un court-circuit de duréc insignifiante, on munit alors celui-ci d'un retardateur réglable. On a alors un disjoncteur temporisé, dont le fonctionnement n'aura licu que si l'excès du débil se maintient pendant un temps déterminé. Ces dis. joncteurs sont parfois appolés disjoncteurs à temps différé, ou disjoncleurs differrés, on, simplomenl, disjoncleur's à temps.

Ces disjoncleurs sonl, cn général, conslilués par

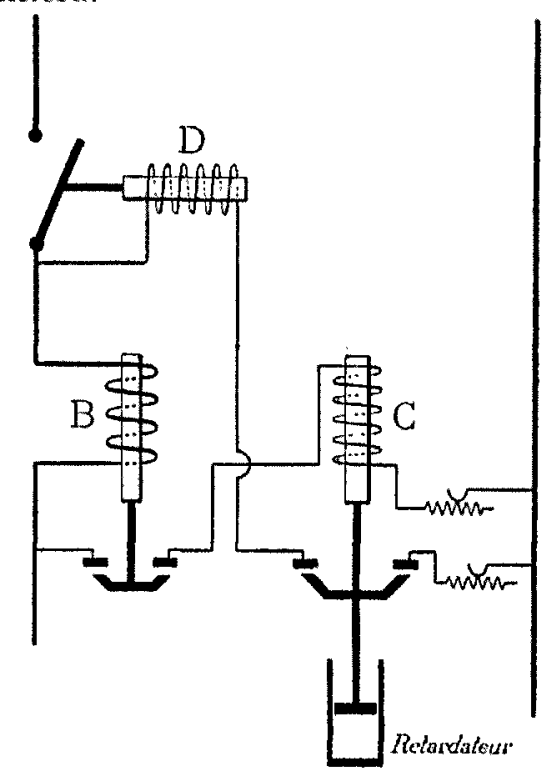

Fı. 13. - Disjoncteur lemporisé. trois bobines (fig. $x 3$ ), la première $B$ regoil le comant princi pal el est réglable à maxima, elle ferme au moment de la sutcharge le circuit de la bobine $C$, dont l'armalure est munie d'un retardateur. Celle bobine fail fermer ì son tour, an bout de la temporisation, le eircuit de la bobine de déclanchement $D$. La durée du relard est réglable soil a l'aide d'unc valve pour un retardateur a air, soil par un coron et 111 contre-ćcrou pour un retardalcur a huile de glycóline.

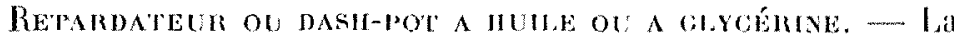
Ggure it représente la coupe d'un retadaleur à glycórine.

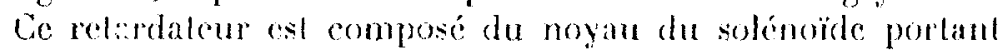

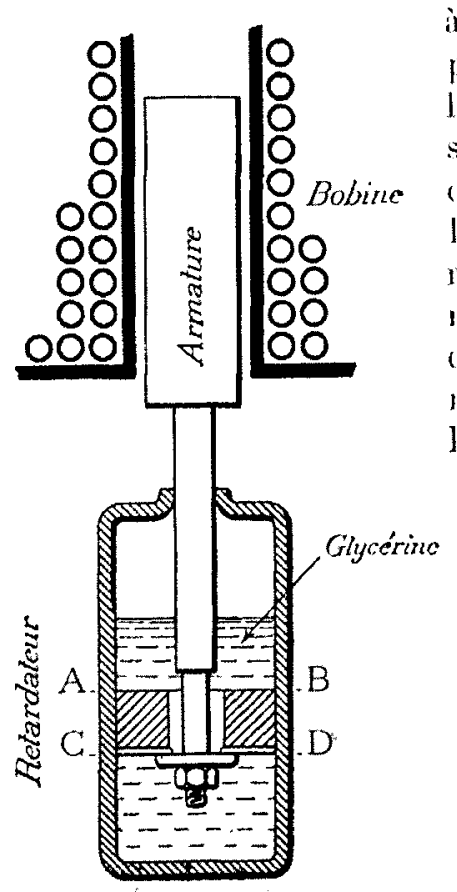

Fig. 14. - Relardateur.

Supposons que le piston soil au bas de sa course d que lc noyau monte par suile du passage du courant dans l'enroulement. La rondelle s'applique, alors contre les orifices a sa patrlis inferiente un pistim ponvant se monvoir dans un cylindre. Le piston a un certain jen sur sa lige of est percé an eentre de qualre orilices débouchant sur les deux faces et à la partic inféricure de qualre pelites minures radiales. Ia tige polte mone romdelle mainlenme pite un écrou, rondelle qui vient sapplicpuer sur les orifices.
Vue en plan suivant $A B$.

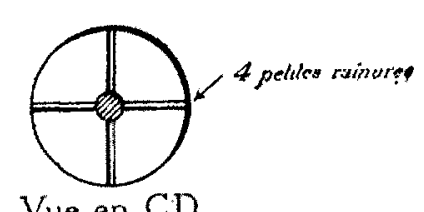

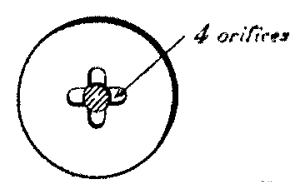


et la glycérine nc peut passer de la partic supérieure à la partic inférieure que par les quatre petiles rainures. Dès que te courant est coupé dans l'enroulement, le noyau descend par son poids, la lige de l'armature appuie sur le piston par son embase, la rondelle se trouve alors à une distance des orifices égale au jou du piston sur la tige; les trous étant complètement débouchis, la glycérine passe rapidement d: la partie inférieure à la partie supérieure du piston, et l'ap. pareil est de nouveau prêt à fonctionner.

Disjoxcteur a action rerarbée. -... Dans ces appareils, on introduit un retard dans le fonctionnenent, mais un relard qui n'est pas fixé à l'avance d'une manière immuable. Ce retard est d'autant moins grand que l'augmentation d'intensité est plus forte. En général, il est obtenu au moyen d'une calaracte mise directement sur le noyau du solénoide.

Un disjoncteur, par exemple, sera réglé pour déclanches à 200 ampères au bout de 10 secondes, cela voudra dire qu'à r go ampères, la rupture ne se produira jamais et qu'à 2oo ampères, elle mettra 10 " pour se produire. Si l'intensité monte jusqu'à 250 ampères, le disjoncteur fonctionnera plus rapidement, après 6" par exemple.

Avec les disjoncteurs relardés, la précision est forcément moins grande qu'avec les disjoncteurs temporisés.

D'une façon générale, les disjoncteurs temporisés peuvent être réglés pour déchancher avec une intensité hrès voisine de l'intensité normale, par' exemple, roo ampères de déclanchement pour go ampèr's (intensité normale), et les disjoncteurs retardés pour déclancher à une intensité bearcoup plus éloignée de l'intensité normale, 250 amp. pour 150 amp., par exemple.

\section{Conclusions}

Il faut apponter beaucoup de soin dans le choix d'un disjoncleur el celui qui en est chargé doit, non seulement posséder parfaitement les caractéristiques des différents dis-. joncleurs, mais savoir, exaclencnt, ce qu'il dósire réaliscr. Dans le cas, par exomple, d'une génératrice chargeant unc batterie d'accumulateurs, il faudra protéger la génćratrice conlre un retour possible de courant ; on pourrait placer un disjoncteur à minima simple sur le circuit de charge, mais nous avons vu que le courant s'inversant brusquement, un lel disjoncteur peut rester enclanché ; on pourrait mettre, également, un disjoncteur à minima el à relour de courant, la généralrice est bien alors protégéc contre un retour. mais elle ne l'est pas contre un excès de courant. Dans le cas d'une seule génératrice, on placerait, sur le circuit de celleci un disjoncteur à minima et a retour. Si on avait plusieurs génératrices en parallèle, par économie, on mettrait un maxima sur chacune d'clles, et un minima polarisé sur le circuil de charge de la balterie.

Chaque cas nécessitc donc une étude spéciale ; étude rapidement faite, d'ailleurs, avec un peu de methode et d'expérience.

Nous n'avons pas la prétention d'avoir éludié tous les cas qui peuvent se presonter dans la pratique, non... loin de là, mais nous serions heureux d'avoir fait ouvre utile envers nos camarades, actuellement, à l'Feole ou dans la carrière industriclle, en leur donnant une idée générale de ces ap. pareils.

Notre but serait complètement alteint, si leur altention élait portée vers des publications de ce genre, publicalions qui seraient insérées dans les bulletins des Socićlés d'Anciens Elèves de l'Institut ou des Arts ot Méliers, ces Notes seraient certainement lues avec plaisir et elles enrichiraient la litté- ralure technique française, si pauere sur l'appareillage éles. Irique.

Nous ne saurions terniner sans remercied Messieurs $B_{A \mathrm{k}}$ BHLON of Bengeor, les distingués Directeur et Sous-1)irerteur, de l'Institut Electrotechnique de Grenoble, qui ont bien voulu lire et revoir notre travail el a qui nous devols cctle représentation graphique éveilléc en nous par celle qu'ils savent employer dans les cours qu'ils professent à nos jeunes camarades.

V. Syluestre. Ingénieur I..E.G.

\section{APPLICATIONS DE L'ÉLECTRICITÉ}

\section{EMPLOI DES LAMPES A VAPEUR DE: MERCURE comme redresseurs de courants alternatifs}

\section{GÉNÉRALITÉs}

La lampe à vapeur de mercure à calhode mercuriclle el à anode constituce par du fer ou du graphite ne peul se laisser lraverser par le courant électrique que dans un seul sens, de l'anode a la cathode el, pour faire fonctionner cetle lampo sur du courant alternatif, il faut avoir recours à des dispositifs particuliers.

Getle curieuse proprićlé, qui conslitue un des inconviniunts de la lampe à mercure dans ses applicalions à l'éclairage, devient un avantage au point de vue de la transformalion possible des courants allernatifs en courant continus.

On sait toule limportance qu'ont les courants allemalifs dans le transporl de l'énergie à grande distance el combien est faible le rendement des appareils utilisés aujourd'hui pour les transformer en courants continus.

Certaines industries ont absolument bosoin do ces derniers courants pour effectuer des opérations spéciales (chargo des accumulateurs, galvanoplastie, électrochimie) ; il importe donc de posséder des appareils ayant à la fois un grand rendement el un prix de revient peu élevé, avec le minimum d'encombrement.

Or, aujourd'hui, celte transformation s'effeclue à l'aide de commulatrices, de permulatrices ou de moteurs génécaleurs, et linstallation de ces machines, d'un prix assez élevé, n'est pratique que lorsqu'il sagit de grandes cxploitations. Les soupapes électrolytiques, employécs aussi depuis quelques années pour les applications n'exigeant qu'une faible puissance, semblent convenir dans un grand nombre de cas; mais leur installation est moins simple ol moins pratique que celle d'une lampe ì mercure qu'il suflit de connecter sur un réseau d'une facon converable pour résondre lo problème.

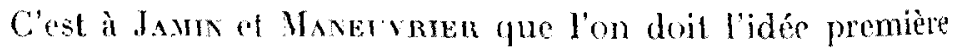
do la transformation possible, sans conmmulateur, des courants alternatifs en courants contimus. Des $188 \%$, ils purent constater que le courant électrique passe plus faciloment à travers un arc lorsqu'il va du gros charbon au petit ou d'une tige de cuive à la surface d'un bain de mercure.la solution pratique de celte conslatation consiste esenticllement ì inlerposer, dans lo circuil exléricur d'une dynamo, un arc voltaïque dissymétrique, cest-it-dire formé entre deux électrodes hétérogènes, soit du charbon el du mercure par exemple.

Ces expériences sont reslées pendant longlemps sans application industriclle importante of ce n'est que dans ces 